\title{
Envolvimento dos hormônios tireoidianos no desenvolvimento e progressão da Doença de Alzheimer
}

\author{
Involvement of thyroid hormones in the development and \\ progression of Alzheimer's Disease
}

\author{
Participación de hormonas tiroides en el desarrollo y \\ progresión de la Enfermedad de Alzheimer
}

Caroline de Sousa dos Reis ${ }^{1}$, Julia Loewen Savaris ${ }^{2}$, Camila Moraes Marques ${ }^{3}$

1.Acadêmica do oitavo período de medicina das Faculdades Pequeno Príncipe (FPP), Curitiba-PR, Brasil. https://orcid.org/0000-0003-4647-4239

2.Acadêmica do oitavo período de medicina das Faculdades Pequeno Príncipe (FPP), Curitiba-PR, Brasil. https://orcid.org/0000-0003-3737-9790

3.Doutora em Ciências Biológicas: Fisiologia e professora das Faculdades Pequeno Príncipe (FPP), CuritibaPR, Brasil. https://orcid.org/0000-0001-5121-922X

\begin{abstract}
Resumo
Introdução. Os hormônios tireoidianos (HTs) são relatados como fatores associados às doenças neurodegenerativas, como a doença de Alzheimer (DA). Objetivo. Analisar a relação entre os hormônios tireoidianos na prevenção e desenvolvimento da doença de Alzheimer. Método. Trata-se de uma revisão integrativa, a partir do levantamento de informações nas bases de dados Science Direct e PubMed. As estratégias de busca estabelecidas foram fundamentais em suas combinações, na língua inglesa, para garantir busca mais ampla. Quanto aos critérios de inclusão para a seleção de artigos: artigos completos e disponíveis, publicados em inglês, entre o período de 2009 até 2020. Artigos incompletos e não disponíveis, publicados anteriormente ao ano de 2009, em outra língua que não o inglês, e artigos redundantes e que não apresentavam relação com o tema, foram excluídos. Resultados. $\mathrm{A}$ glândula tireoide e seus diversos distúrbios possuem íntima relação no funcionamento fisiológico e patológico cerebral, podendo estar relacionado ao desenvolvimento, progressão e tratamento de doenças neurodegenerativas, especialmente com a doença de Alzheimer (DA), apesar das atuais evidências ainda não serem claras. Conclusão. São necessárias mais pesquisas para desvendar o elo fisiopatológico causal exato entre a função da tireoide e a demência. Definir mecanismos fisiopatológicos causais não apenas fornece mais informações sobre o desenvolvimento da demência, como também pode descobrir possíveis triagens futuras ou novas opções de tratamento.
\end{abstract}

Unitermos. Doença de Alzheimer; Hormônios tireóideos; Hipertireoidismo; Hipotireoidismo; Receptores dos hormônios tireóideos

\footnotetext{
Abstract

Introduction. Thyroid hormones (HTs) are reported as factors associated with neurodegenerative diseases, such as Alzheimer's disease (AD). Objective. To analyze the relationship between thyroid hormones in the prevention and development of Alzheimer's disease. Method. It is an integrative review, based on the collection of information in the Science Direct and PubMed databases. The established search strategies were fundamental in their combinations, in the English language, to guarantee a broader search. As for the inclusion criteria for the selection of articles: complete and available articles, published in English, from 2009 to 2020. Incomplete and unavailable articles published before 2009, in a language other than English, and redundant articles that were not related to the topic were excluded. Results. The thyroid gland and its various disorders are closely related to brain physiological and pathological functioning, and may be related to the development, progression, and treatment of neurodegenerative diseases, especially with Alzheimer's disease (AD), although the current
} 
evidence is still unclear. Conclusion. Further research is needed to unravel the exact causal pathophysiological link between thyroid function and dementia. Defining causal pathophysiological mechanisms not only provides more information about the development of dementia but can also discover possible future screening or new treatment options.

Keywords. Alzheimer's disease; Thyroid hormones; Hyperthyroidism; Hypothyroidism; Thyroid hormone receptors

\begin{abstract}
Resumen
Introducción. Las hormonas tiroideas (HT) se informan como factores asociados con enfermedades neurodegenerativas, como la enfermedad de Alzheimer (EA). Objetivo. Analizar la relación entre las hormonas tiroideas en la prevención y el desarrollo de la enfermedad de Alzheimer. Método. Es una revisión integradora, basada en la recopilación de información en las bases de datos Science Direct y PubMed. Las estrategias de búsqueda establecidas fueron fundamentales en sus combinaciones, en el idioma inglés, para garantizar una búsqueda más amplia. En cuanto a los criterios de inclusión para la selección de artículos: artículos completos y disponibles, publicados en inglés, de 2009 a 2020. Artículos incompletos y no disponibles, publicados antes de 2009, en un idioma que no sea inglés, y se excluyeron los artículos redundantes que no estaban relacionados con el tema. Resultados La glándula tiroides y sus diversos trastornos están estrechamente relacionados con el funcionamiento fisiológico y patológico del cerebro, que puede estar relacionado con el desarrollo, la progresión y el tratamiento de enfermedades neurodegenerativas, especialmente con la enfermedad de Alzheimer (EA), aunque la evidencia actual aún no está clara. Conclusión. Se necesita más investigación para desentrañar el vínculo fisiopatológico causal exacto entre la función tiroidea y la demencia. La definición de los mecanismos fisiopatológicos causales no solo proporciona más información sobre el desarrollo de la demencia, sino que también puede descubrir posibles exámenes de detección futuros o nuevas opciones de tratamiento.
\end{abstract}

Palabras clave. Enfermedad de Alzheimer; Hormonas tiroideas; Hipertiroidismo; Hipotiroidismo; Receptores de hormona tiroidea

Trabalho realizado na Faculdades Pequeno Príncipe (FPP), Curitiba-PR, Brasil.

Conflito de interesse: não Recebido em: 10/07/2020 Aceito em: 31/08/2020

Endereço de correspondência: Camila M Marques. R Alferes Ângelo Sampaio 2765, Bairro Bigorrilho, Curitiba-PR, Brasil. CEP 80730-460. E-mail: profcamilafpp@gmail.com

\title{
INTRODUÇÃO
}

A tireoide é uma glândula endócrina cuja função é regulada pelo hormônio liberador de tirotrofina (TRH) produzido no hipotálamo, que se dirige à adeno-hipófise e induz a síntese e secreção de hormônio tirotrófico (TSH) e este, por sua vez, induz a biossíntese de HTs, predominantemente de tiroxina (T4), pró-hormônio precursor, por meio da desiodação pelas enzimas desiodase 
1 (D1) e 2 (D2), da maior parte do tri-iodotironina (T3) circulante ${ }^{1}$. Já a desiodase 3 (D3) é muito expressa no SNC em desenvolvimento e está envolvida com a inativação dos $\mathrm{HTs}^{2}$. Além de T3 e T4, a tireoide também secreta calcitonina, hormônio atuante no metabolismo do cálcio. Do T3, a forma ativa do hormônio, é que depende a atividade de, praticamente, todos os tecidos do organismo, seu crescimento, diferenciação, regulação da atividade $\mathrm{e}$ metabolismo, visto que todos eles, potencialmente, expressam receptores de $\mathrm{HTs}^{3}$.

Seus efeitos biológicos sistêmicos são desencadeados pela interação com receptores nucleares (TR) e, consequente, modulação positiva ou negativa da transcrição de genes-alvo, assim, promovendo ou não a síntese de proteínas específicas ${ }^{2}$. Para o T3 ser capaz de reprimir a transcrição de um gene, deve haver ligação do TR em uma região promotora específica que contenha negative T3response elemento (nTRE), onde se iniciaria a transcrição, e, assim como na modulação positiva, através da ligação com um elemento de resposta à tireoide (TRE), promover e estabilizar o TR junto ao promotor do gene $e^{4}$. Há um grande número de genes regulados por T3, porém, seu efeito não se restringe a esta ação descrita, podendo também afetar o mecanismo de transcrição através de modificações de cromatina, como por metilação do DNA, o convertendo de eucromatina para heterocromatina, e reduzindo a metilação e acetilação de histonas ${ }^{4}$. 
Para alcançar as células neurais, T4 e T3 precisam atravessar a barreira hematoencefálica (BHE), um processo facilitado por transportadores específicos: transportador mono carboxilato 8 (MCT8) para T4 e T3, e o polipeptídeo transportador de ânion orgânico 1C1 (OATP1C1) para T4², para, então, agir em seus receptores nucleares cerebrais TRa e TR $\beta$, constituindo 9 isoformas (TRa1, a2, a3, $\Delta a 1, \Delta a 2, \beta 1$, $\beta 2$, $\beta 3$ e $\Delta \beta 3$ ), sendo que TRa1 e TRß2 são os de principal funcionalidade no cérebro ${ }^{5}$.

A atuação fisiológica dos HTs e seus distúrbios interferem diretamente no desenvolvimento e funcionamento cerebral, com maturação de neurônios específicos, proliferação, migração e diferenciação celular, sinaptogênese, mielinização e sinalização, influenciam também no humor, comportamento e localização axonal de mapas cerebrais ${ }^{6}$. Desde a década de 60 é observado uma relação entre as disfunções tireoidianas e sintomas neurológicos durante o envelhecimento, como a depressão, irritabilidade, psicose, comprometimento da memória, ataxia cerebelar e tremulação?.

Tanto 0 hipo quanto 0 hipertireoidismo são considerados causas de comprometimento cognitivo ${ }^{8}$, assim como, igualmente, tanto as patologias evidentes, quanto as subclínica, ou seja, com TSH alterado e T4 em níveis normais ${ }^{9}$, e variações dentro do intervalo normal, foram associados com risco aumentado de fenótipos associados à idade e mortalidade ${ }^{3}$. Estudos experimentais sugerem uma sensibilidade particular do hipocampo, uma região-chave da 
aprendizagem e memória, para disfunção tireoidiana neonatal e adulta 9 .

Neste mesmo aspecto, os HTs são relatados como fatores associados às doença neurodegenerativas, incluindo a $D A^{10}$, doença caracterizada por morte neuronal excessiva e prematura em regiões focais do córtex, resultando em atrofia focal das áreas afetadas do cérebro ${ }^{10}$. A DA é uma DN, sendo a causa mais comum de demência diagnosticada após os 60 anos, acometendo cerca de $1 \%$ da população entre 65 e 69 anos, quinta causa de morte em pessoas na mesma faixa etária e predominante em mulheres ${ }^{5}$. Resistência à insulina, cortisol elevado, baixo estrogênio e todos os níveis de testosterona foram implicados no desenvolvimento e/ou progressão da DA. Entretanto, a associação mais amplamente reconhecida envolve os $\mathrm{HTs}^{7}$. As características neuropatológicas dos cérebros com DA incluem depósitos fibrilares amiloidais localizados nas paredes dos vasos sanguíneos, uma variedade de diferentes tipos de placas senis, principalmente do peptídeo $\beta$-amiloide $(A \beta)$, formada pelo processamento, pelas enzimas secretases, da proteína precursora de amiloide (APP), cuja expressão gera estresse oxidativo, que, então, leva a neurotoxicidade, apoptose neuronal e perda sináptica, lesão do sistema colinérgico do SNC e danos ao DNA- hipótese inflamatória. Há também acúmulo de filamentos anormais da proteína tau e consequente formação de novelos neurofibrilares (NFT) ${ }^{11}$. Além disso, também há disfunção mitocondrial dos neurônios, gerando depleção da reserva de 
ATP cerebral, prejudicando as funções neuronais e privação de fatores de crescimento, hipótese mitocondrial ${ }^{11}$. A demência é sua forma de manifestação, caracterizada pela perda progressiva da função cognitiva e memória. Os principais sinais da doença são dificuldade em lembrar de conversas, eventos ou nomes. Apatia e depressão são sinais primários; posteriormente, surgem comprometimento da comunicação, mau julgamento, confusão, desorientação, mudanças de comportamento e, eventualmente, dificuldade em engolir, falar e caminhada não suave ${ }^{5}$.

Esta pesquisa tem como objetivo discutir os possíveis mecanismos pelos quais os HTs estão associados no desenvolvimento da DA, resumindo diversos estudos recentes sobre o tema. Segundo o Instituto Oswaldo Cruz, a incidência de doenças neurodegenerativas tem aumentado significativamente nos últimos anos, tornando-as um dos maiores desafios atuais da saúde pública. Prevê-se que cerca de $20 \%$ da população mundial terá 60 anos ou mais até $2050^{12}$, ou seja, a incidência destas doenças será ainda maior.

\section{MÉTODO}

O estudo trata-se de uma revisão integrativa, a partir do levantamento de informações nas bases de dados Science Direct e PubMed. Para a seleção dos artigos, foram estabelecidos os seguintes Descritores em Ciências da Saúde (DeCS): Thyroid Hormones AND Alzheimer's disease OR 
Neurodegenerative diseases AND Cognitive Decline. As estratégias de buscas estabelecidas foram fundamentais em suas combinações em diferentes formas, na língua inglesa, para garantir uma busca mais ampla. Quanto a critérios de inclusão para a seleção de artigos: artigos completos e disponíveis, publicados em inglês, entre o período de 2009 até 2020. Os critérios de exclusão foram: artigos não completos e não disponíveis, publicados anteriormente ao ano de 2009, em outra língua que não o inglês, e artigos redundantes e que não apresentavam relação com o tema.

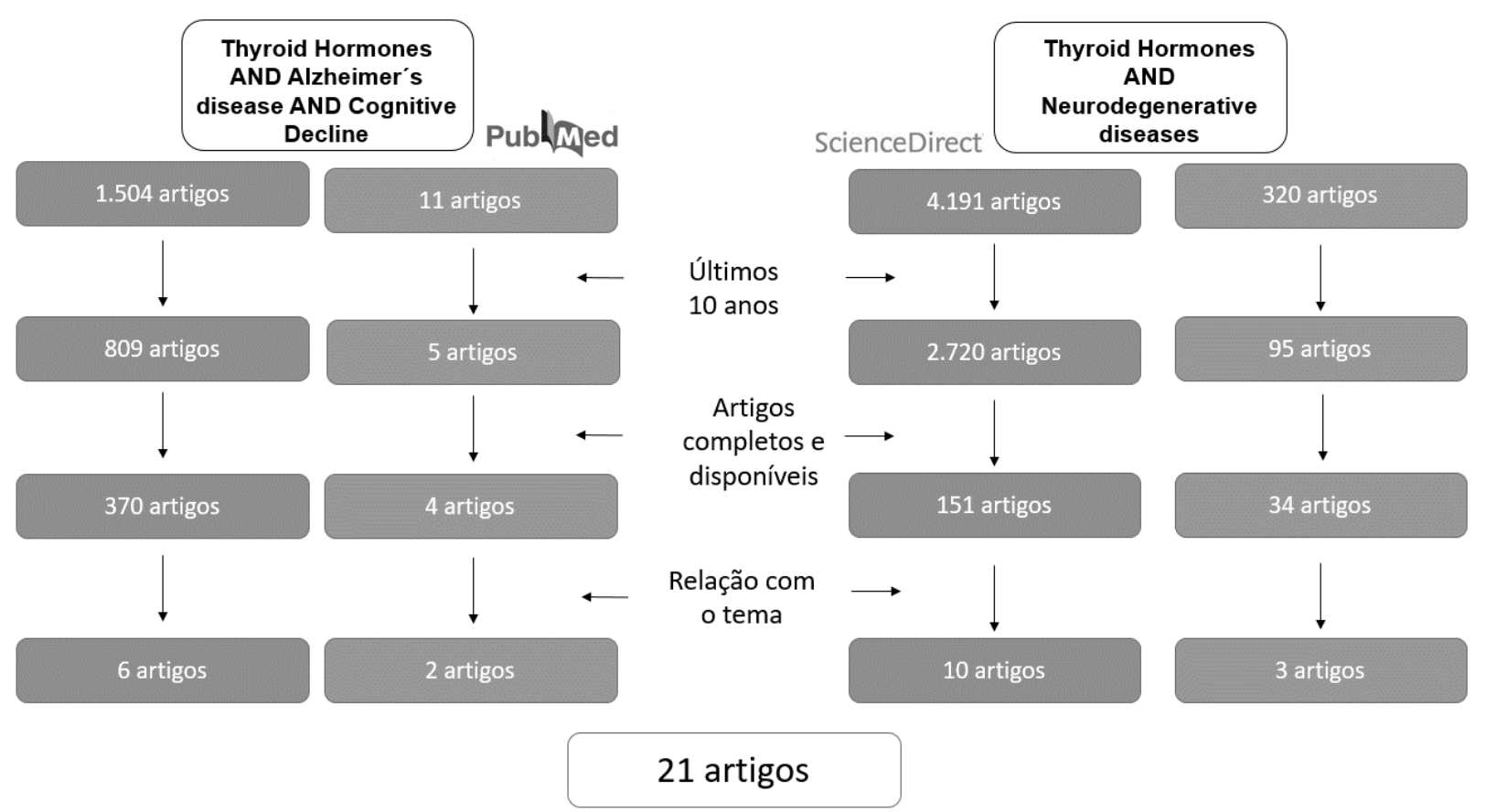




\section{RESULTADOS e DISCUSSÃO}

\section{Hormônios tireoidianos e a fisiopatogênese da DA}

Hormônios tireoidianos e proteína ApoE

No cérebro existem proteínas chamadas apolipoproteína E (ApoE), sintetizadas e secretadas por astrócitos, microglia e, em menor grau, por neurônios ${ }^{13}$, geralmente envolvidas no metabolismo lipídico, em 3 diferentes subtipos: ApoE2, ApoE3 e ApoE4, cada uma expressa quantitativamente de forma diferente em cada indivíduo e apresentando suas peculiaridades na influência do funcionamento do cérebro ${ }^{14,15}$.

Cérebros com maior expressão de ApoE2, fato mais comum em homens, possuem esta proteína como forma de proteção cerebral contra o desenvolvimento da DA, pois, em sua presença, há menor deposição de $A \beta$ e formação de NTF, além de modular melhor a sinalização por IgF1, o metabolismo da glicose e o domínio catalítico da H+ -ATPase do tipo $V$ que concentra neurotransmissores nas vesículas sinápticas ${ }^{13}$. Também é responsável por reparar lesões através da redistribuição de lipídios entre os neurônios, modular o crescimento de neurites e da integridade cerebrovascular ${ }^{14}$. A ApoE3 é considerada neutra quanto em proteger ou propiciar o desenvolvimento da $D A^{13}$. Já a ApoE4, mais comum em mulheres, é relacionada a taxa acelerada e gravidade aumentada do declínio cognitivo, com menor início de idade da doença e resposta alterada aos tratamentos. Este subtipo contribui para a hiperfosforilação da proteína tau, etapa importante no desenvolvimento da 
$\mathrm{DA}^{8}$. Em seu predomínio, há também maior atrofia cerebral, menor metabolismo da glicose, função sináptica e neurogênese de hipocampo. Esses cérebros são menos eficientes na depuração de $A \beta$, por menor quantidade e afinidade da proteína pelo metabólito ${ }^{13}$.

Em relação aos HTs, o T3 é capaz de aumentar a expressão de ApoE2, no cérebro, pelos astrócitos, em até 50 vezes $^{6}$. Faz isso aumentando a transcrição do gene da ApoE2, através da ligação do TR ativado por ligante com o promotor seletivo ME. $2^{6}$. A expressão de ApoE2, aumentada pelo T3, induz à reexpressão da subunidade NR2B do receptor N-metil-D-aspartato (NMDA), auxiliando na recuperação da memória e aprendizado prejudicados por doenças cognitivas. $O$ próprio processo de envelhecimento demonstrou estar associado a um déficit na NR2B ${ }^{14}$.

Hormônios tireoidianos e APP

Os HTs são capazes também de alterar um ponto fundamental da fisiopatogênese da $D A$, o acúmulo de placas de $A \beta$. $O T 3$, em sua função de regulação gênica, regula negativamente a atividade transcricional do gene de APP, pela ligação do TR nuclear no 10 éxon do gene, diminuindo os níveis intracelulares do RNA mensageiro (mRNA) da APP, do processamento e secreção de APP em si e, consequentemente, de $A \beta^{4,7,10}$ ou seja, no hipotireoidismo, há aumento da expressão de APP, então, de $A \beta$ e maiores chances de desenvolver $\mathrm{DA}^{8}$. 
Da mesma forma, somente na presença de T3, proteínas chamadas HDAC e HKDM são recrutadas para o promotor do APP, onde facilitam a repressão do gene ${ }^{4}$. Estudos demonstraram níveis aumentados de mRNA e APP no hipocampo, mas não no cerebelo, de ratos hipotireoidianos, revertido com a administração de $\mathrm{T} 4^{10}$. Baixos níveis séricos de fT4, mesmo no estado clínico da tireoide, foram associados a um aumento na carga cerebral de $A \beta^{16}$.

Hormônios tireoidianos e risco vascular cerebral

Os níveis alterados de HTs, principalmente de TSH, também são relacionados a aumento do risco vascular, como fibrilação atrial e hipertensão sistólica, assim, podendo afetar indiretamente 0 risco de DA por danos cerebrais vasculares $7,8,17$.

Hormônios tireoidianos e energia cerebral

Os HTs influenciam também no metabolismo energético cerebral: T4 aumenta a produção de ATP cerebral no hipocampo e córtex frontal, por aumentar a biogênese mitocondrial' ${ }^{11}$. No hipotireoidismo, há interferência na manutenção da energia normal da glicose, consumida em processos necessários para funções cerebrais essenciais, como neurotransmissão e memória ${ }^{5}$. Níveis séricos de TSH aumentados e baixos de Ft4 podem estar relacionados a hipometabolismo local da glicose ${ }^{16}$. 
Hormônios tireoidianos e estresse oxidativo

Já citado anteriormente, na fisiopatogênese da DA, existe a hipótese inflamatória, em que o acúmulo de placas $A \beta$ gera estresse oxidativo. Neste aspecto, tanto o hipo quanto 0 hipertireoidismo possuem influência. Já no desenvolvimento embrionário, o nível de expressão do óxido nítrico sintetase neuronal (nNOS) e da atividade enzimática é regulado pela glândula tireóide 5 .

No hipotireoidismo, T4 aumenta o nível de glutamato desidrogenase $(\mathrm{GDH})$, uma enzima anti-oxidante, por aumento de sua transcrição, então, diminuindo o estresse oxidativo induzido por $A \beta$, prevenindo a apoptose neural, especialmente de neurônios colinérgicos, e aumentando a atividade da ChAT e liberação de Ach, diminuídos na DA ${ }^{11}$.

$O$ hipertireoidismo pode afetar o estresse oxidativo por aumentar a taxa basal metabólica do consumo doe oxigênio, produzindo mais espécies reativas de oxigênio e menor atividade de metabólitos anti-oxidantes ${ }^{7,18}$, alterando a integridade neural e podendo gerar morte celular ${ }^{18}$.

Estudos mostram que, com o aumento de T4, são encontrados mais casos de demência subclínica gerada por esta inflamação ${ }^{8}$. Lesões por radicais livres são associados a alterações da substância branca cerebral ${ }^{18}$, seja micro ou macroestrutural. Níveis mais altos de fT4 são associados a maiores volumes cerebrais e aumento da integridade da substância branca, mas não cinzenta, em jovens, enquanto maiores níveis de TSH são associados a menores volumes cerebrais de substância branca, mas não volume de 
substância cinzenta, também em pacientes jovens. Esses achados podem indicar um efeito dependente da idade de HTs na morfologia cerebral e na organização microestrutural, benéfico em idade mais jovem e deletério em idade mais avançada $^{18}$.

Hormônios tireoidianos e neuroserpina

Na fisiologia, o plasminogênio é convertido em plasmina pelo seu ativador tPA, com principal função na coagulação sanguínea. Em forma de plasmina, exerce também a função de depurar $A \beta$ e degradar APP. A proteína neuroserpina é capaz de inibir a ativação do plasminogênio pelo seu ativador, que, por si só, aumenta a atividade sináptica no hipocampo. Nos cérebros DA, há aumento da expressão de neuroserpina e HUd, proteína que atua póstranscricionalmente para melhorar a expressão de inúmeras proteínas, incluindo da neuroserpina, estabilizando seu mRNA $^{19}$.

Níveis aumentados patológicos de HTs aumentam a expressão de neuroserpina, por meio do aumento da expressão da proteína HuD, que, então, estabiliza seu mRNA, melhorando sua tradução e aumentando seus níveis nos tecidos ${ }^{19}$.

Portanto, em cérebros DA, há relação positiva significativa entre neuroserpina e THRb e neuroserpina e $\operatorname{HuD}^{19}$. 
Hormônios tireoidianos e fator neurotrófico derivado do cérebro

Há no cérebro, com expressão neuroanatômica mais alta em hipocampo e no córtex, proteínas chamadas de reelina, da matriz extracelular, e o fator neurotrófico derivado do cérebro (BDNF) ${ }^{15}$. Segundo estudos, a proteína BDNF possui a função de reprimir a expressão de $A \beta$, regulação da transmissão sináptica e da plasticidade neuronal ${ }^{15}$, enquanto a reelina diminui a formação e agregação das placas de $A \beta$ e sua neurotoxicidade, e aumenta plasticidade sináptica ${ }^{16,15}$. Além disso, esta proteína regula a fosforilação, a expressão das subunidades do receptor NMDA, já citada sua importância e relação com os HTs, facilita o influxo de Ca2+ e estimula a via de sinalização intracelular envolvida na potenciação de longo prazo por implicações diretas nas subunidades NMDAR ${ }^{15}$. Justamente, ambas as proteínas estão em concentrações diminuídas em cérebros de indivíduos com $D A$, e que a morte neuronal induzida por $A \beta$ pode ser uma consequência da deficiência de BDNF. Portanto, uma super-expressão de reelina em populações de neurônios melhorou o número de sinapses no hipocampo ${ }^{15}$.

A administração de T3 intra-hipocampal é capaz de aumentar a expressão de BDNF e reelina no hipocampo de ratos com DA. A redução dos níveis séricos de T4, T3 e FT4 reduziu a expressão do mRNA e da proteína do BDNF no hipotálamo em ratos machos. Além disso, o tratamento pósnatal temporário de ratos com T4 e T3 aumentou os níveis de proteína BDNF e mRNA desta no hipocampo ${ }^{15}$. 


\section{Hipotireoidismo e doença de Alzheimer}

O hipotireoidismo é considerado uma causa comum de demência reversível. A disfunção tireoidiana subclínica é ainda mais comum e também tem sido implicado no declínio da função cognitiva em idosos ${ }^{20}$. Esta alteração é relacionada a baixa captação de glicose, alterando a neurotransmissão, memória e funções cerebrais ${ }^{12}$.

Um estudo transversal de base populacional monofásica, com participantes de 65 anos, totalizando em 1.276 idosos, demonstrou associação positiva entre hipotireoidismo subclínico, demência e DA, em homens ${ }^{21}$. Da mesma forma, foi encontrada associação positiva entre hipotireoidismo e comprometimento cognitivo em uma amostra de pacientes idosos, bem como uma associação positiva entre os participantes com níveis elevados de FT4 normais e comprometimento cognitivo após acompanhamento de dois anos ${ }^{21}$. Assim como o hipotireoidismo pode induzir à DA, esta pode diminuir os níveis de TRH, TSH e T4, induzindo a progressão da própria doença ${ }^{8}$.

Em relação a níveis cerebrais dos HTs, três estudos constataram o mesmo: o córtex pré-frontal de pacientes com características clínicas da DA podem estar em um estado de tecido hipotireóideo ${ }^{15}$, assim como, no estudo de casocontrole, foram encontrados níveis mais baixos de T3 e TSH em pacientes com DA grave em comparação com participantes controle ${ }^{7}$, e relatórios de laboratório 
mostraram que a DII e os níveis de T3 diminuíram no cérebro de ratos com $\mathrm{DA}^{15}$.

Além disso, uma diminuição no nível de T4 e T3 dentro da faixa padrão é um forte preditor de alterações cognitivas em mulheres ${ }^{15}$. Mulheres com níveis de T4 mais baixos tiveram um duplo risco de declínio cognitivo em um período de 3 anos, em comparação com as mulheres com maiores níveis, em estudo comparando 464 mulheres idosas ${ }^{9}$.

Em um pequeno estudo, demonstrou-se disfunção cognitiva em 54 pacientes que apresentaram melhora após 5 meses de terapia tireóidea ${ }^{9}$. Prova disso é, a administração de T4 restaurou a aprendizagem e memória de camundongos com características de DA induzida por injeção de $A \beta^{11}$.

Além disso, um estudo, analisando 5 formas de polimorfismos no nucleotídeo do TRa, assim, desenvolvendo resistência aos HTs, demonstrou que os participantes portadores da mutação possuem risco maior de desenvolver a $\mathrm{DA}^{22}$.

\section{Hipertireoidismo e doença de Alzheimer}

O hipertireoidismo também está implicado como fator de risco para desenvolvimento da DA na população idosa ${ }^{8}$. Níveis mais altos de T4 e total estão consistentemente associados a demência e $\mathrm{DA}^{21}$. Até mesmo em níveis elevados normais, há comprometimento cognitivo após acompanhamento de dois anos ${ }^{21}$. 
A doença em sua forma subclínica é associada a um risco 3x maior de $\mathrm{DA}^{7}$, encontrada, em estudo, relação somente em homens ${ }^{21}$.

A patologia é relacionada a ruptura do sistema colinérgico e ao estresse oxidativo sistêmico ${ }^{12}$.

O estudo de Roterdã demonstrou que níveis elevados de HTs são associados a atrofia cerebral em idosos não dementados, atrofia principalmente hipocampal e amigdaliana, em ressonância ${ }^{21,23}$.

\section{CONCLUSÃO}

O artigo demonstra a complexidade potencial da relação entre os HTs e a DA, propiciando melhor entendimento da fisiopatologia, tratamento e prevenção da demência. O enfoque no tratamento da doença tireoidiana, mesmo que subclínica, pode ser importante para diminuir a incidência da doença. Todavia, o tratamento excessivo pode se tornar mais prevalente, e os efeitos da função tireoidiana exógena no risco de demência são semelhantes aos da tireoide endógena ainda precisa ser determinado.

\section{REFERÊNCIAS}

1. Molina PE. Fisiologia Endócrina - 2. Porto Alegre: AMGH Editora. 2009.

2.Bernal J. New insights on thyroid hormone and the brain. Curr Opin Endocr Metabol Res 2018;2:24-8.

https://doi.org/10.1016/j.coemr.2017.12.001 
3. Quinlan $P$, Horvath A, Wallin A, Svensson J. Low serum concentration of free triiodothyronine (FT3) is associated with increased risk of Alzheimer's disease. Psychoneuroendocrinol 2019;99:112-9.

https://doi.org/10.1016/j.psyneuen.2018.09.002

4.Belakavadi M, Dell J, Grover GJ, Fondell JD. Thyroid hormone suppression of $\beta$-amyloid precursor protein gene expression in the brain involves multiple epigenetic regulatory events. Mol Cell Endocrinol 2011;339:72-80.

https://doi.org/10.1016/j.mce.2011.03.016

5.Bavarsad K, Hosseini M, Hadjzadeh MAR, Sahebkar A. The effects of thyroid hormones on memory impairment and Alzheimer's disease. J Cell Physiol 2019;234:14633-40. https://doi.org/10.1002/jcp.28198 6. Roman C, Fuior EV, Trusca VG, Kardassis D, Simionescu M, Gafencu AV. Thyroid hormones upregulate apolipoprotein $\mathrm{E}$ gene expression in astrocytes. Bioch Biophys Res Commun 2015;468:190-5. https://doi.org/10.1016/j.bbrc.2015.10.132

7.Tan ZS, Vasan RS. Thyroid function and Alzheimer's disease. J Alzheimers Dis 2009;16:503-7. http://doi.org/10.3233/JAD-20090991

8.Toro CA, Zhang L, Cao J, Cai D. Sex differences in Alzheimer's disease: Understanding the molecular impact. Brain Res 2019;1719:194-207. https://doi.org/10.1016/j.brainres.2019.05.031 9. Etgen $\mathrm{T}$, Bickel $\mathrm{H}$, Förstl $\mathrm{H}$. Metabolic and endocrine factors in mild cognitive impairment. Ageing Res Rev 2010;9:280-8. https://doi.org/10.1016/j.arr.2010.01.003

10.Contreras-Jurado C, Pascual A. Thyroid hormone regulation of APP ( $\beta$-amyloid precursor protein) gene expression in brain and brain cultured cells. Neurochemistr Int 2012;60:484-7. https://doi.org/10.1016/j. neuint.2012.01.027

11.Fu AL, Zhou CY, Chen X. Thyroid hormone prevents cognitive deficit in a mouse model of Alzheimer's disease. Neuropharmacol 2010;58:722-9. https://doi.org/10.1016/j.neuropharm.2009.12.020

12.Soni M, White LR, Kridawati A, Bandelow S, Hogervorst E. Phytoestrogen consumption and risk for cognitive decline and dementia: with consideration of thyroid status and other possible mediators. J Steroid Biochemistr Mol Biol 2016;160:67-77. https://doi.org/10.1016/j.jsbmb.2015.10.024

13. Wu L, Zhao L. ApoE2 and Alzheimer's disease: time to take a closer look. Neural Regen Res 2016;11:412-3.

https://doi.org/10.4103/1673-5374.179044

14. Moncayo R, Ortner K. Multifactorial determinants of cognitionthyroid function is not the only one. BBA Clin 2015;3:289-98. https://doi.org/10.1016/j.bbacli.2015.04.002

15.Shabani S, Farbood Y, Sarkaki A, Mard SA, Ahangarpour A, Khorsandi L. The effect of triiodothyronine on the hippocampal longterm potentiation in an animal model of the Alzheimer's disease: The role of BDNF and reelin. Neurol Psychiatr Brain Res 2019;33:82-8. https://doi.org/10.1016/j.npbr.2019.07.004 
16. Choi HJ, Byun MS, Yi D, Sohn BK, Lee JH, Lee J, et al. Associations of thyroid hormone serum levels with in-vivo Alzheimer's disease pathologies. Alzheimer's Res Therap 2017;9:1-9.

https://doi.org/10.1186/s13195-017-0291-5

17. Chaker L, Wolters FJ, Bos D, Korevaar TI, Hofman A, van der Lugt $A$, et al. Thyroid function and the risk of dementia: The Rotterdam Study. Neurology 2016;87:1688-95.

https://doi.org/10.1212/WNL.0000000000003227

18. Chaker L, Cremers LG, Korevaar TI, De Groot M, Dehghan A, Franco $\mathrm{OH}$, et al. Age-dependent association of thyroid function with brain morphology and microstructural organization: evidence from brain imaging. Neurobiol Aging 2018;61:44-51.

https://doi.org/10.1016/j.neurobiolaging.2017.09.014

19.Subhadra B, Schaller K, Seeds NW. Neuroserpin up-regulation in the Alzheimer's disease brain is associated with elevated thyroid hormone receptor- $\beta 1$ and HuD expression. Neurochemistr Int 2013;63:476-81. https://doi.org/10.1016/j.neuint.2013.08.010

20.Ojala AK, Schalin-Jäntti C, Pitkälä KH, Tilvis RS, Strandberg TE. Serum thyroid-stimulating hormone and cognition in older people. Age Ageing 2016;45:155-7. https://doi.org/10.1093/ageing/afv160

21.Benseñor IM, Lotufo PA, Menezes PR, Scazufca M. Subclinical hyperthyroidism and dementia: the Sao Paulo ageing \& Health study (SPAH). BMC Public Health 2010;10:1-8.

https://doi.org/10.1186/1471-2458-10-298

22.Goumidi L, Flamant F, Lendon C, Galimberti D, Pasquier F, Scarpini $\mathrm{E}$, et al. Study of thyroid hormone receptor alpha gene polymorphisms on Alzheimer's disease. Neurobiol Aging 2011;32:624-30. https://doi.org/10.1016/j. neurobiolaging.2009.04.007

23.de Jong FJ, Masaki K, Chen H, Remaley AT, Breteler MM, Petrovitch $\mathrm{H}$, et al. Thyroid function, the risk of dementia and neuropathologic changes: The Honolulu-Asia Aging Study. Neurobiol Aging 2009;30:600-6.

https://doi.org/10.1016/j.neurobiolaging.2007.07.019 